\title{
Erratum to: An Unsupervised Text-Mining Approach and a Hybrid Methodology to Improve Early Warnings in Construction Project Management
}

\author{
Mohammed Alsubaey, Ahmad Asadi and Charalampos Makatsoris
}

\section{Erratum to:}

"An Unsupervised Text-Mining Approach and a Hybrid Methodology to Improve Early Warnings in Construction Project Management" in: Y. Bi et al. (eds.), Intelligent Systems and Applications, Studies in Computational Intelligence 650, DOI 10.1007/978-3-319-33386-1_4

The chapter was inadvertently published without including the updates in the affiliations, a change in the corresponding author's name from Harris Makatsoris to Prof. Charalampos Makatsoris and contact, also some more additional corrections within the chapter. The original chapter has been updated with the changes.

The updated original online version for this chapter can be found at DOI 10.1007/978-3-319-33386-1_4

M. Alsubaey · A. Asadi

Department of Mechanical, Aerospace and Civil Engineering,

Brunel University, London, UK

e-mail: alsubaey.mohammed@gmail.com
A. Asadi
e-mail: AhmadAsadi@outlook.com
C. Makatsoris $(\square)$

School of Aerospace, Transportation and Manufacturing,

Sustainable Manufacturing Systems Centre, Cranfield University,

Bedfordshire MK43 0AL, UK

e-mail: h.makatsoris@cranfield.ac.uk 